\title{
Seguimiento a la aplicación del programa de estudios de la MIEEB
}

\section{Follow up on the implementation of the program of the MIEEB studies}

\author{
DÁVILA-GUTIÉRREZ，Alicia†*，LÓPEZ-CANO，Xóchitl，LÓPEZ-VICTORIANO, Mariana y \\ MONROY-PLATA, Isabel
}

Escuela Normal de Ixtlahuaca. Av. Emiliano Zapata s/n, Santo Domingo de Guzmán, 50740 Ixtlahuaca de Rayón, Méx.

ID $1^{\text {er }}$ Autor: Alicia, Dávila-Gutiérrez / ORC ID: 0000-0001-6591-0468, CVU CONACYT ID: 476923

ID 1 ${ }^{\text {er }}$ Coautor: Xóchitl, López-Cano / ORC ID: 0000-0002-0885-5092, CVU CONACYT ID: 947239

ID $2^{\text {do }}$ Coautor: Mariana, López-Victoriano / ORC ID: 0000-0002-3921-3340, CVU CONACYT ID: 84407

ID $3^{\text {er }}$ Coautor: Isabel, Monroy-Plata / ORC ID: 0000-0002-7739-6301, CVU CONACYT ID: 947238

DOI: $10.35429 / J U P .2019 .10 .3 .1 .13$

Recibido 02 de Octubre, 2019; Aceptado 27 de Diciembre, 2019

\section{Resumen}

El Seguimiento a la aplicación del Programa de Estudios de la Maestría en Intervención Educativa para la Educación Básica (MIEEB), en la Escuela Normal de Ixtlahuaca (ENI) se realiza a partir de diversos procedimientos, momentos y funciones, tales como: el "monitoreo y evaluación a la aplicación de planes y programas de estudio de posgrado" del Sistema de gestión de Calidad (SGC), otro es, el seguimiento que se efectúa desde la función del Coordinador de Posgrado. El objetivo de estos procedimientos es: "obtener información sobre los avances e incidencias de Programas Educativos de Posgrado que se ofertan en las Escuelas Normales, para coadyuvar en el aseguramiento de la calidad educativa de los mismos" (SEN, 2017), y el de este reporte, es reflexionar en los resultados de la aplicación del Programa de Estudios de la MIEEB: fortalezas y debilidades. La metodología de este estudio es a partir del análisis de documentos que han sido aplicados a los estudiantes y docentes, los que se han emitido para informar a las autoridades escolares y educativas. Se pretende contribuir a la reflexión de los alcances que tiene el programa de maestría en la profesionalización de los docentes de Educación Básica.

Seguimiento, Maestría, Reflexión

\begin{abstract}
The Follow-up to the application of the Program of Studies of the Master in Educational Intervention for Basic Education (MIEEB), in the Normal School of Ixtlahuaca (ENI) is being made from various procedures, moments and functions, such as: the procedure "monitoring and evaluation to the application of plans and programs of postgraduate study" of the Quality Management System (QMS), another is the follow-up that is carried out from the position of the Postgraduate Coordinator. The objective of these procedures is: "Obtain information on the progress and incidences of Postgraduate Educational Programs that are offered in the Normal Schools, to help in the assurance of their educational quality "(SEN, 2017), and the one in this report, is to reflect on the results of the application of the MIEEB Program of Studies: strengths and weaknesses. The methodology of this study is based on the analysis of documents that have been administered to students and teachers, those that have been issued to inform school and educational authorities. It is intended to contribute to the reflection of the scope of the master's program in the professionalization of teachers of Basic Education.
\end{abstract}

Follow-Up, Master's, Reflection

Citación: DÁVILA-GUTIÉRREZ, Alicia, LÓPEZ-CANO, Xóchitl, LÓPEZ-VICTORIANO, Mariana y MONROYPLATA, Isabel. Seguimiento a la aplicación del programa de estudios de la MIEEB. Revista de Políticas Universitarias. 2019. 3-10: 1-13.

\footnotetext{
* Correspondencia del Autor (Correo electrónico: alice1380@yahoo.com.mx)

$\dagger$ Investigador contribuyendo como primer autor.
} 


\section{Introducción}

En la Escuela Normal de Ixtlahuaca se implementó la Maestría en Intervención Educativa para la Educación Básica (MIEEB), promoción 2017-2019, y para cumplir con el propósito formativo: "Profesionalizar a los docentes, fortaleciendo sus competencias en Intervención Educativa, concretada en el diseño de prototipos didácticos, y sustentada en la Investigación-Acción, para responder a las demandas sociales y a las políticas de evaluación en pro de la calidad de la educación" (SEN, 2017), se elaboró un Plan de Trabajo con aspectos solicitados por la Subdirección de Educación Normal, el objetivo de este plan es: "obtener información sobre los avances e incidencias de Programas Educativos de Posgrado que se ofertan en las Escuelas Normales, para coadyuvar en el aseguramiento de la calidad educativa de los mismos".

Uno de los procesos de atención de este plan de trabajo es el Seguimiento a la aplicación de Plan y Programas de Estudio, el cual está vinculado a otros, tales como, dinámica del núcleo docente en la aplicación de Plan y Programas de Estudio; atención al modelo, enfoque; orientación del Programa Educativo y modalidad educativa; seguimiento a la trayectoria estudiantil. La vinculación existente entre ellos permite, a partir del primero, realizar el seguimiento a los demás, por tanto, es importante que los instrumentos que se apliquen para tal seguimiento sean diversos.

Así, en el presente escrito se analizará el proceso Seguimiento a la aplicación de Plan y Programas de Estudio recuperando la información desde diversos instrumentos, tiempos de aplicación y funciones. Los asuntos y procesos de atención referidos en el plan de trabajo se recuperan en el informe del Seguimiento Académico de los Posgrados que se ofertan en las Escuelas Normales Públicas del Estado de México (SEN, 2017). Por tanto, la pregunta de esta investigación, es: ¿cuál es el impacto del seguimiento a la aplicación de plan y programas de estudio en la profesionalización de los docentes de Educación Básica? La hipótesis que se plantea es: el seguimiento la aplicación de planes y programas de estudio incide en su cumplimiento y en logro del perfil de egreso de la maestría.

\section{Metodología}

En la MIEEB el seguimiento a la aplicación los Planes y Programas de Estudio fue considerado un aspecto fundamental para la reflexión y la toma de decisiones y se realizó a partir de los siguientes procesos, procedimientos e instrumentos: Seguimiento Académico de los Posgrados; Valoración de los cursos; Proceso de Seguimiento a la Aplicación del Planes y Programas de Estudio del Sistema de Gestión de la Calidad; Reporte de avance del prototipo didáctico; Valoración de los cursos; Valoración del Prototipo Didáctico; Estudio de Factibilidad, este último fue aplicado por única vez a los estudiantes de la maestría con el propósito de evaluar el Programa de la MIEEB y realizar la Adecuación. Los seis primeros han sido sistemáticos y se efectúan durante o al término de cada cuatrimestre, los resultados fueron socializados al inicio de cuatrimestre en la Jornada de Planeación y Actualización a los docentes (asesores y tutores) para conocer las fortalezas y debilidades en la aplicación del programa y establecer acuerdos que lo fortalezcan.

El Seguimiento Académico de los Posgrados se realizó a partir de un formato diseñado por los responsables del posgrado de la Subdirección de Educación Normal (SEN), el cual remitió el responsable del programa educativo, los elementos variaron durante los cuatrimestres en los que fue solicitado, para el IV Cuatrimestre se solicitó informar la dinámica del núcleo docente en la aplicación del Plan y Programas de Estudio (ver Tabla 1).

\section{Seguimiento y valoración al proceso operativo} del plan de estudios

a. Dinámica del núcleo docente en la aplicación de Plan y Programas de Estudio

- Operación de las asignaturas/cursos u otras opciones.

Didácticas que se utilizan para el desarrollo de los cursos.

Nivel de dominio de los espacios curriculares por el núcleo docente, desde el inicio hasta el momento actual.

Dudas, preguntas o cuestionamientos sobre el plan de estudios por parte de docentes y estudiantes.

- Desarrollo de procesos de investigación.

- $\quad$ Manejo y gradualidad de desarrollo de la malla curricular.

Tabla 1 Aspectos del Seguimiento Académico de los Posgrados 
En el Seguimiento Académico de los Posgrados, se resalta la importancia de incluir una metodología, criterios e indicadores reconocidos. La Metodología de CIEES 2014, propone criterios que se vinculan a los emitidos en las orientaciones para elaborar el plan de trabajo y los informes (ver Tabla 2).

\section{Métodos de enseñanza-aprendizaje}

Efectividad de los métodos empleados en la formación integral de los estudiantes, en particular en:

a) congruencia con el modelo educativo;

b) función del perfil de egreso que se pretende;

c) adecuación a los objetivos del plan de estudios;

d) efectividad para el cumplimiento de los contenidos y los objetivos de cada asignatura;

e) congruencia con la naturaleza teórica-práctica de cada asignatura;

f) fines del plan de estudios en términos de los aprendizajes genéricos que habrá de lograr el alumno;

g) correspondencia de las actividades de aprendizaje con los objetivos del programa;

h) adecuación de las actividades de aprendizaje a la modalidad educativa;

i) adecuación del número promedio de estudiantes que atiende cada profesor.

Tabla 2

La Metodología CIEES, orienta la autoevaluación de los programas educativos con el propósito evaluarlos y certificarlos. Por tanto, algunos de estos criterios son considerados para el seguimiento del programa de maestría con el propósito de recuperar la información de cómo se está implementando el programa y su impacto en la profesionalización docente.

El instrumento Valoración de los cursos, se aplicó para recuperar la información con relación a implementación de los cursos y realizar el rediseño curricular.

Los componentes del programa que se valoraron son: unidades, contenidos, líneas de discusión, competencias didácticas, producto integrador, evaluación, fuentes de consulta; se agregó un apartado para observaciones y sugerencias. La valoración enfatizó en especificar del componente: la página, la modificación y la sugerencia. También constituyó un instrumento de seguimiento porque valoró el conocimiento que el docente tuvo del curso (ver Tabla 3).

\begin{tabular}{l|l|l|l|}
\hline $\begin{array}{l}\text { Componente Dice/página } \\
\text { Mel programa }\end{array}$ Modificación & Sugerencias \\
\hline Unidades & & & \\
\hline Contenidos & & & \\
\hline $\begin{array}{l}\text { Líneas de } \\
\text { discusión }\end{array}$ & & & \\
\hline Competencias & & & \\
\hline $\begin{array}{l}\text { Producto } \\
\text { integrador }\end{array}$ & & & \\
\hline Evaluación & & & \\
\hline $\begin{array}{l}\text { Fuentes de } \\
\text { consulta }\end{array}$ & & & \\
\hline Otros & & & \\
\hline Observaciones generales & \\
\hline
\end{tabular}

Tabla 3 Instrumento para la valoración de los cursos

En el Sistema de Gestión de Calidad (SGC), implementado en las Escuelas Normales del estado de México, a partir del ciclo escolar 2017-2018 se declaró como auditables a los procedimientos académicos: Elaboración de la planificación docente de asignatura o curso y, Monitoreo y evaluación de la aplicación de la planificación docente de asignatura o curso. El segundo procedimiento lo realizó el responsable del seguimiento a la aplicación del plan y programa de estudios de la maestría con las siguientes acciones: observación en el aula, reporte de avance del prototipo didáctico (emitido por los estudiantes) y la encuesta de satisfacción del cliente.

La observación en el aula se realizó a partir de la guía de observación. Esta guía da seguimiento a la aplicación de los programas de curso a partir de dos rubros: observable o no observable en los siguientes ejes: se presenta el contenido de la sesión, se presentan las líneas de discusión del contenido; se promueve la reflexión a partir de las líneas de discusión; se desarrollan las actividades vinculadas al contenido y las líneas de discusión; se favorece una modalidad didáctica acorde al contenido; se promueve la participación argumentada; el producto académico que se genera está vinculado al contenido y líneas de discusión de la sesión; se favorece la articulación del contenido con los programas de educación básica y/o la investigación educativa (ver Tabla 4). 


\begin{tabular}{|c|c|c|c|}
\hline Eje & $\begin{array}{c}\text { Se } \\
\text { observa }\end{array}$ & $\begin{array}{c}\text { No se } \\
\text { observa }\end{array}$ & Observaciones \\
\hline $\begin{array}{l}\text { 1. Se presenta el } \\
\text { contenido de } \\
\text { la sesión. }\end{array}$ & & & \\
\hline $\begin{array}{l}\text { 2.Se presentan } \\
\text { las líneas de } \\
\text { discusión del } \\
\text { contenido. }\end{array}$ & & & \\
\hline $\begin{array}{l}\text { 3. Se promueve } \\
\text { la reflexión a } \\
\text { partir de las } \\
\text { líneas de } \\
\text { discusión. }\end{array}$ & & & \\
\hline $\begin{array}{l}\text { 4.Se } \\
\text { desarrollan } \\
\text { las } \\
\text { actividades } \\
\text { vinculadas al } \\
\text { contenido y } \\
\text { líneas de } \\
\text { discusión. }\end{array}$ & & & \\
\hline $\begin{array}{l}\text { 5.Se favorece } \\
\text { una } \\
\text { modalidad } \\
\text { didáctica } \\
\text { acorde al } \\
\text { contenido. }\end{array}$ & & & \\
\hline $\begin{array}{l}\text { 6. Se promueve } \\
\text { la } \\
\text { participación } \\
\text { argumentada. }\end{array}$ & & & \\
\hline $\begin{array}{l}\text { 7.El producto } \\
\text { académico } \\
\text { que se genera } \\
\text { está } \\
\text { vinculado al } \\
\text { contenido y } \\
\text { las líneas de } \\
\text { discusión de } \\
\text { la sesión. }\end{array}$ & & & \\
\hline $\begin{array}{l}\text { 8. Se favorece } \\
\text { la } \\
\text { articulación } \\
\text { del contenido } \\
\text { con los } \\
\text { programas de } \\
\text { educación } \\
\text { básica y/o la } \\
\text { investigación } \\
\text { educativa. }\end{array}$ & & & \\
\hline
\end{tabular}

Tabla 4 Guía de observación

El reporte de avance del prototipo didáctico se realizó por parte del estudiante que valoró en términos de porcentaje su avance en la construcción de su documento de grado, el instrumento tiene como elementos: nombre del docente, asesorados, avance reportado y porcentaje de avance (ver Tabla 5).

\begin{tabular}{|c|c|c|c|}
\hline $\begin{array}{c}\text { Nombre } \\
\text { del } \\
\text { docente }\end{array}$ & Asesorados & $\begin{array}{c}\text { Avance } \\
\text { reportado }\end{array}$ & $\begin{array}{c}\% \\
\text { avance }\end{array}$ \\
\hline & & & \\
\hline
\end{tabular}

Tabla 5 Reporte de avance del prototipo didáctico

La encuesta de satisfacción del cliente se aplicó al término de cada cuatrimestre y valora el grado de satisfacción del estudiante con relación a cuatro aspectos: la planificación docente, el logro del propósito del curso, el cumplimiento del propósito formativo del programa de estudios $\mathrm{y}$, el dominio del contenido por parte del titular del curso (ver Tabla 6).

\begin{tabular}{|c|c|c|c|c|}
\hline Rubro & $\begin{array}{c}\text { Muy } \\
\text { satisfecho }\end{array}$ & Satisfecho & $\begin{array}{c}\text { Poco } \\
\text { satisfecho }\end{array}$ & $\begin{array}{l}\text { Insatisf } \\
\text { echo }\end{array}$ \\
\hline $\begin{array}{l}\text { Aplica la } \\
\text { planificación } \\
\text { docente del } \\
\text { curso de } \\
\text { acuerdo a los } \\
\text { criterios } \\
\text { establecidos al } \\
\text { inicio de } \\
\text { cuatrimestre }\end{array}$ & & & & \\
\hline $\begin{array}{l}\text { Cumple con el } \\
\text { Programa de } \\
\text { Estudios } \\
\text { logrando los } \\
\text { propósitos del } \\
\text { curso }\end{array}$ & & & & \\
\hline $\begin{array}{l}\text { Cumple con el } \\
\text { propósito } \\
\text { formativo del } \\
\text { Programa de } \\
\text { Estudios }\end{array}$ & & & & \\
\hline $\begin{array}{l}\text { El titular } \\
\text { muestra } \\
\text { dominio del } \\
\text { contenido } \\
\text { programático } \\
\text { del curso }\end{array}$ & & & & \\
\hline
\end{tabular}

Tabla 6 Encuesta de satisfacción del cliente

La coordinación de la maestría aplicó distintos instrumentos de seguimiento al plan y programas de estudio y esto se efectuó desde el primer cuatrimestre en el que aún no se habían declarado los procedimientos del SGC para tal efecto.

El que a continuación se presenta realiza el seguimiento al programa de curso, la conducción por parte del docente y la participación del estudiante, este instrumento fue aplicado al principio a estudiantes y docentes y a partir del IV cuatrimestre sólo a los estudiantes (ver Tabla 7). 


\begin{tabular}{|c|c|c|c|}
\hline $\begin{array}{l}\text { El propósito del } \\
\text { curso se logró }\end{array}$ & $\begin{array}{l}\text { I. } \\
\text { Insuficiente } \\
\text { (s) }\end{array}$ & $\begin{array}{l}\text { Regular } \\
\text { (es) }\end{array}$ & Totalmente \\
\hline $\begin{array}{l}\text { Los contenidos del } \\
\text { curso fueron }\end{array}$ & & & \\
\hline $\begin{array}{l}\text { El desarrollo de los } \\
\text { contenidos del curso } \\
\text { fue }\end{array}$ & & & \\
\hline $\begin{array}{l}\text { Los contenidos del } \\
\text { curso se vinculan a } \\
\text { la práctica docente }\end{array}$ & & & \\
\hline $\begin{array}{l}\text { Los contenidos del } \\
\text { curso se vinculan } \\
\text { con otros cursos }\end{array}$ & & & \\
\hline $\begin{array}{l}\text { Las competencias } \\
\text { docentes descritas } \\
\text { en el curso fueron } \\
\text { desarrolladas }\end{array}$ & & & \\
\hline $\begin{array}{l}\text { El producto final } \\
\text { está vinculado a los } \\
\text { contenidos del curso }\end{array}$ & & & \\
\hline $\begin{array}{l}\text { Los contenidos del } \\
\text { curso impactan en la } \\
\text { profesionalización } \\
\text { docente }\end{array}$ & & & \\
\hline $\begin{array}{l}\text { El producto final es } \\
\text { de calidad y se } \\
\text { puede incorporar a } \\
\text { la Tesis de grado }\end{array}$ & & & \\
\hline $\begin{array}{l}\text { Las competencias } \\
\text { del curso impactan } \\
\text { en el logro del Perfil } \\
\text { de Egreso }\end{array}$ & & & \\
\hline
\end{tabular}

Tabla 7 Valoración de cursos

Otro instrumento de seguimiento al plan y programas de la maestría es el cuestionario que se empleó para conocer el avance en la construcción del prototipo didáctico, este fue aplicado a los docentes al finalizar cada cuatrimestre y en él se solicitó valorar el estado de los componentes del documento para la obtención de grado y realizar observaciones al respecto.

Los componentes corresponden a la estructura de la tesis y se integran los correspondientes al cuatrimestre cursado (ver Tabla 8).

\begin{tabular}{|c|c|c|c|c|}
\hline Componente & $\begin{array}{c}\text { Est } \\
\text { Inicial }\end{array}$ & $\begin{array}{c}\text { do del com } \\
\begin{array}{c}\text { En } \\
\text { proceso }\end{array}\end{array}$ & $\begin{array}{l}\text { ponente } \\
\text { Concluido }\end{array}$ & $\begin{array}{c}\text { Observaciones } \\
\text { (especifique } \\
\text { las fortalezas } \\
\text { v deblilidades) }\end{array}$ \\
\hline $\begin{array}{l}\text { Título } \\
\text { Se especifica el } \\
\text { tipo de Prototipo } \\
\text { Didáctico y está } \\
\text { integrado por las } \\
\text { categorías } \\
\text { principales. }\end{array}$ & & & & \\
\hline $\begin{array}{l}\text { Diagnóstico } \\
\text { Se analiza la } \\
\text { problemática de } \\
\text { la práctica } \\
\text { docente a partir } \\
\text { de las } \\
\text { dimensiones: } \\
\text { personal, } \\
\text { interpersonal, } \\
\text { didáctica, social, } \\
\text { institucional y } \\
\text { valorar. }\end{array}$ & & & & \\
\hline $\begin{array}{lr}\text { Planteamiento } \\
\text { del problema } \\
\text { Se especifica } \\
\text { cual es el } \\
\text { problema } \\
\text { intervenir } \\
\text { partir a } \\
\text { identificar sus } \\
\text { causas y efectos. } \\
\end{array}$ & & & & \\
\hline $\begin{array}{l}\text { Justificación } \\
\text { Se analiza la } \\
\text { importancia de } \\
\text { intervenir el } \\
\text { problema y cuál } \\
\text { es } \\
\text { consecuencia de } \\
\text { no hacerlo. }\end{array}$ & & & & \\
\hline $\begin{array}{ll}\text { Pregunta de de } & \text { de } \\
\text { investigación } \\
\text { Se plantea la } \\
\text { pregunta } & \text { en } \\
\text { términos } & \text { de } \\
\text { intervención. }\end{array}$ & & & & \\
\hline
\end{tabular}

Tabla 8 Valoración del Prototipo Didáctico

El instrumento que se empleó para realizar la adecuación del Programa de la MIEEB es el cuestionario Estudio de Factibilidad, el cual, también proporcionó información de la aplicación del programa. El instrumento fue elaborado por la comisión interinstitucional y las preguntas valoran los propósitos cursos y las competencias del perfil de egreso (ver Tabla 9). 


\begin{tabular}{|c|c|c|c|}
\hline $\begin{array}{l}\text { De qué manera ha } \\
\text { contribuido la MIEEB } \\
\text { en los siguientes } \\
\text { aspectos: }\end{array}$ & Suficiente & Poco & $\begin{array}{l}\text { No ha } \\
\text { influido }\end{array}$ \\
\hline $\begin{array}{l}\text { Analizar, reflexionar y } \\
\text { transformar la práctica } \\
\text { educativa atendiendo la } \\
\text { complejidad y el } \\
\text { contexto del trabajo en la } \\
\text { educación básica. }\end{array}$ & & & \\
\hline $\begin{array}{l}\text { Producir conocimientos } \\
\text { para la mejora continua } \\
\text { en la intervención hacia } \\
\text { una práctica educativa de } \\
\text { calidad a partir de } \\
\text { procesos } \\
\text { investigación. }\end{array}$ & & & \\
\hline $\begin{array}{l}\text { Desarrollar su identidad } \\
\text { profesional y ética } \\
\text { atendiendo asertiva y } \\
\text { creativamente } \\
\text { problemáticas las } \\
\text { necesidades inherentes a } \\
\text { su práctica educativa. }\end{array}$ & & & \\
\hline $\begin{array}{l}\text { Los fundamentos } \\
\text { teóricos, metodológicos } \\
\text { y didácticos } \\
\text { contemplados en los } \\
\text { cursos: didáctica de las } \\
\text { matemáticas, español y } \\
\text { ciencias han permitido: }\end{array}$ & & & \\
\hline $\begin{array}{lcr}\text { Fortalecer } & & \text { el } \\
\text { conocimiento de } & \text { la } \\
\text { didáctica para } & \text { la } \\
\text { enseñanza y } & \text { el } \\
\text { aprendizaje, en el diseño } \\
\text { de situaciones } & \text { de } \\
\text { aprendizaje y } & \text { su } \\
\text { evaluación. } & & \end{array}$ & & & \\
\hline
\end{tabular}

Tabla 9 Estudio de factibilidad

El seguimiento a la aplicación del Programa de Estudios de la MIEEB se realizó durante los seis cuatrimestres que duró la maestría y los instrumentos empleados permiten triangular la información de tal manera que los resultados con relación al impacto del programa en la profesionalización docente se constatan.

\section{Resultados}

Los procedimientos e instrumentos de seguimiento referidos permitieron detectar las fortalezas y debilidades tanto en la aplicación de los programas de curso como en su diseño. Derivado del análisis del Seguimiento Académico de los Posgrados, se elaboraron conclusiones y sugerencias con relación a los ejes correspondientes (ver Tabla 10).

\section{Conclusiones}

Operación de las asignaturas/cursos u otras opciones

La operación de los programas de asignatura ha sido apegada al mismo, se desarrollan los contenidos a partir de las líneas de discusión, se sustenta en la bibliografía propuesta y se agregan referentes que fortalezcan esta operatividad.

Didácticas que se utilizan para el desarrollo de los cursos

La didáctica está fundamentada en las líneas de discusión, por tanto, se fomenta la argumentación, el diálogo, la reflexión, se vinculan los contenidos con los programas de Educación Básica y con la construcción del Prototipo Didáctico; se favorece el trabajo en equipo, la investigación, la lectura, la escritura de textos.

Nivel de dominio de los espacios curriculares por el núcleo docente, desde el inicio hasta el momento actual

El nivel de dominio que tienen los docentes la maestría es valorado por los estudiantes como bueno, los cursos se asignan de acuerdo al perfil profesional y los docentes realizan una planeación de clase para apoyar su sesión; las observaciones realizadas a las sesiones confirman que éstas son preparadas y los docentes muestran un claro dominio de los contenidos.

Dudas, preguntas o cuestionamientos sobre el plan de estudios por parte de docentes $y$ estudiantes.

Las dudas que está generando el plan de estudios se concentran en el Prototipo Didáctico por la carencia de los programas, y aun cuando se le vincula directamente con el trayecto formativo metodológico, se precisa la concreción de los cursos del trayecto referido, lo que dará certeza en el producto final.

Sugerencias:

- Construcción de los programas de cursos que faltan de la MIEEB, por la comisión de diseño curricular inicial.

- Promover de forma permanente cursos estatales de planeación y capacitación para la implementación de los programas de maestría.

- Integrar los cuerpos académicos estatales de posgrado.

- Favorecer la movilidad de la planta docente de posgrado.

- Disminuir la carga de funciones de los docentes de posgrado.

Tabla 10 IV Informe cuatrimestral del Seguimiento Académico de los Posgrados (diciembre de 2018) 
Diciembre, 2019 Vol.3 No.10 1-13

Se observó acorde con los resultados de los cursos implementados hasta el cuatrimestre IV que estos se desarrollaban de acuerdo con el programa, esto es, se trabajan los contenidos de acuerdo con las líneas de discusión fomentando el diálogo, el análisis, la argumentación y se fortalece con la integración de otros referentes bibliográficos. Los docentes de acuerdo con los resultados tienen buen dominio de los contenidos y una de las causas es la asignación de los cursos de acuerdo a su perfil profesional. Por otra parte, se planteó el análisis de las dudas que generó el plan de estudios y éstas se concretaron en los cursos del Trayecto Formativo Prototipo Didáctico porque aún no tenía definido sus contenidos, el alcance de los cursos y el producto final, aspectos que se consideraron al realizar la Adecuación del Programa de la MIEEB. Uno de los instrumentos que apoyó el informe y la adecuación del programa de la maestría fue el denominado valoración de los cursos, el cual se solicitó a los docentes de curso al finalizar cada cuatrimestre. Esta valoración se concretó en los componentes de los programas de curso y el docente realizó la sugerencia concretando el párrafo y página (ver Tabla 11).

\begin{tabular}{|c|c|c|c|}
\hline $\begin{array}{c}\text { Componente } \\
\text { del } \\
\text { programa }\end{array}$ & Dice/página & Modificación & Sugerencias \\
\hline $\begin{array}{l}\text { Unidades } \\
\text { I Planeación } \\
\text { Didáctica y II } \\
\text { Planeación } \\
\text { Didáctica } \\
\text { Argumentada } \\
\text { - } \\
\text { III Las TAC } \\
\text { en Educación }\end{array}$ & $\begin{array}{l}\text { En las } \\
\text { páginas } 2 \text { y } 3 \\
\text { del plan de } \\
\text { curso } \\
\text { entregado } \\
\text { por el } \\
\text { profesor. } \\
\text { En la página } \\
4 .\end{array}$ & $\begin{array}{l}\text { No sufrieron } \\
\text { modificaciones } \\
\text { y se trabajaron } \\
\text { tal cual fueron } \\
\text { planeadas. } \\
\text { No sufrió } \\
\text { modificaciones }\end{array}$ & $\begin{array}{l}\text { Se sugiere } \\
\text { que estas } \\
\text { unidades } \\
\text { sean } \\
\text { fusionadas } \\
\text { en una sola. } \\
\text { Y se } \\
\text { adicione una } \\
\text { unidad II que } \\
\text { trabaje el } \\
\text { tema de "El } \\
\text { Proyecto de } \\
\text { Enseñanza". } \\
\text { Trabajar ésta } \\
\text { unidad } \\
\text { totalmente } \\
\text { en prácticas } \\
\text { de } \\
\text { laboratorio } \\
\text { de } \\
\text { informática. }\end{array}$ \\
\hline $\begin{array}{l}\text { Contenidos } \\
\text { De la unidad } \\
\text { I y Unidad II }\end{array}$ & $\begin{array}{ll}\text { En las } \\
\text { páginas } 2 \text { y } 3 \\
\text { del plan de } \\
\text { curso } \\
\text { entregado } \\
\text { por } \\
\text { profesor } & \\
\end{array}$ & $\begin{array}{l}\text { No sufrieron } \\
\text { modificaciones }\end{array}$ & $\begin{array}{l}\text { Se sugiere } \\
\text { que estos } \\
\text { contenidos } \\
\text { sean } \\
\text { fusionados } \\
\text { en uno solo. }\end{array}$ \\
\hline $\begin{array}{ll}\text { Líneas } & \mathrm{de} \\
\text { discusión } & \end{array}$ & $\begin{array}{ll}\text { En las } \\
\text { páginas } 2 \text { y } 3 \\
\text { del plan de } \\
\text { curso } & \\
\text { entregado } & \\
\text { por } & \text { el } \\
\text { profesor } & \end{array}$ & $\begin{array}{l}\text { No sufrieron } \\
\text { modificaciones } \\
\text {. }\end{array}$ & $\begin{array}{l}\text { Se sugiere } \\
\text { que estas } \\
\text { líneas sean } \\
\text { reducidas y } \\
\text { fusionadas. }\end{array}$ \\
\hline
\end{tabular}

\begin{tabular}{|c|c|c|c|}
\hline $\begin{array}{l}\text { Competencias } \\
\text { Didácticas }\end{array}$ & $\begin{array}{l}\text { En la Página } \\
1\end{array}$ & $\begin{array}{l}\text { Ninguna } \\
\text { modificación }\end{array}$ & $\begin{array}{l}\text { Ninguna } \\
\text { sugerencia }\end{array}$ \\
\hline $\begin{array}{l}\text { Producto } \\
\text { integrador }\end{array}$ & $\begin{array}{l}\text { En la Página } \\
1\end{array}$ & & $\begin{array}{l}\text { Se sugiere } \\
\text { una } \\
\text { Planeación } \\
\text { Didáctica } \\
\text { Argumentad } \\
\text { a junto con } \\
\text { un Proyecto } \\
\text { de } \\
\text { Enseñanza }\end{array}$ \\
\hline Evaluación & $\begin{array}{l}\text { Se planeó de } \\
\text { la siguiente } \\
\text { manera: Pág. } \\
2 \text { a Pág. } 4 \\
\text { •12 } \\
\text { Cuartillas } \\
\text { y/o Mapas } \\
\text { como } \\
\text { evidencias } \\
\text { conceptuales } \\
\text { • } 20 \% \\
\text { planeación } \\
\text { didáctica } \\
\text { argumentada } \\
\text { unidad II } \\
40 \% \text {. } \\
\text { •Ensayo uso } \\
\text { de las TAC } \\
10 \% \text {. }\end{array}$ & $\begin{array}{l}\text { Se ejecutó de la } \\
\text { siguiente } \\
\text { manera: •10 } \\
\text { Talleres 20\%. } \\
\text { •Planeación } \\
\text { didáctica } \\
\text { unidad I 20\% } \\
\text { •planeación } \\
\text { didáctica } \\
\text { argumentada } \\
\text { evidencia } \\
\text { unidad II } 40 \% \text {. } \\
\text {-Ensayo uso de } \\
\text { las TAC 20\%. }\end{array}$ & \\
\hline $\begin{array}{l}\text { Fuentes de } \\
\text { consulta }\end{array}$ & $\begin{array}{l}\text { En las } \\
\text { páginas } 4 \text { y } 5 \\
\text { del plan de } \\
\text { curso. }\end{array}$ & $\begin{array}{ll}\text { No } & \text { hubo } \\
\text { cambios } & \end{array}$ & $\begin{array}{l}\text { Se sugiere } \\
\text { agregar } \\
\text { autores para } \\
\text { el Proyecto } \\
\text { de } \\
\text { Enseñanza }\end{array}$ \\
\hline
\end{tabular}

Tabla 11 Valoración del curso

La valoración del curso Planeación didáctica argumentada permite recuperar las sugerencias para la adecuación del programa como implementar el proyecto de enseñanza, es la denominación a la planificación en la reforma curricular 2018 para Educación Básica.

Las debilidades detectadas en el diseño de los programas, por los docentes responsables de curso, permitió integrar sugerencias para la Adecuación del Programa de la Maestría. Las sugerencias se concretaron en fortalecer la malla curricular al analizar la pertinencia de los cursos en los distintos cuatrimestres, es decir, algunos cursos tendrían mayor relevancia en un cuatrimestre anterior o posterior al presentado (ver Tabla 12). 


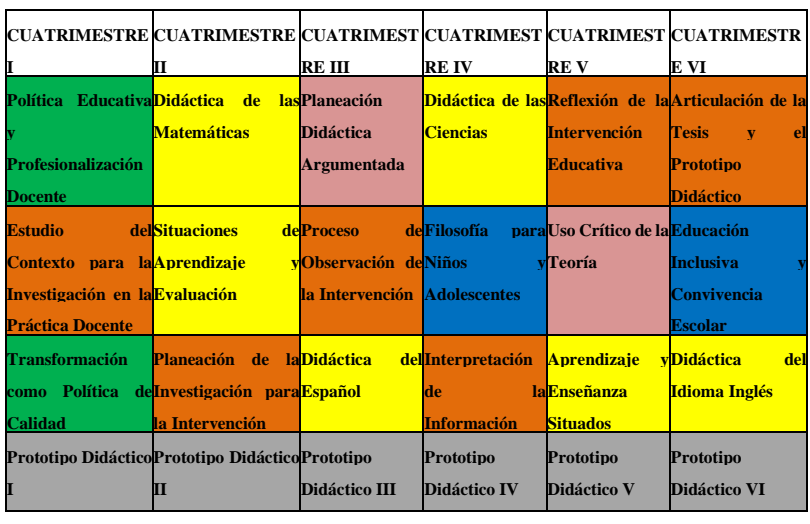

Tabla 12 Malla curricular MIEEB 2017

En el primer cuatrimestre, se ubicaron dos cursos correspondientes al Trayecto Formativo Socio-político, posterior a su implementación y en la adecuación se consideró pertinente ubicar el curso Transformación como Política de Calidad en el segundo cuatrimestre para profundizar en el conocimiento de la política educativa y no agotar su tratamiento en el primer cuatrimestre.

De la misma forma, se integró al primer cuatrimestre el curso del Trayecto Formativo Práctica Educativa: Situaciones de Aprendizaje y Evaluación que estaba en el segundo cuatrimestre. Los siguientes cursos cambiaron de cuatrimestre: Planeación Didáctica Argumentada del tercer al segundo cuatrimestre, Didáctica de las matemáticas del segundo al tercer cuatrimestre (ver Tabla 13).

La malla se fortaleció en el Trayecto Formativo Prototipo Didáctico porque aún no se habían diseñado los programas de curso y tampoco se les había asignado un título.

Los cursos de este trayecto se diseñaron a la par de la implementación de los cuatrimestres por el equipo de tutores y posteriormente se retomó esta experiencia por el equipo de diseño para definir los contenidos y el nombre del curso.

Con relación a los cursos, el que sufrió mayores adecuaciones fue Didáctica de las Ciencias que cambio el enfoque epistémico y con ello, su propósito, unidades, contenidos, bibliografía.

Los demás, se fortalecieron con la integración de referentes bibliográficos.

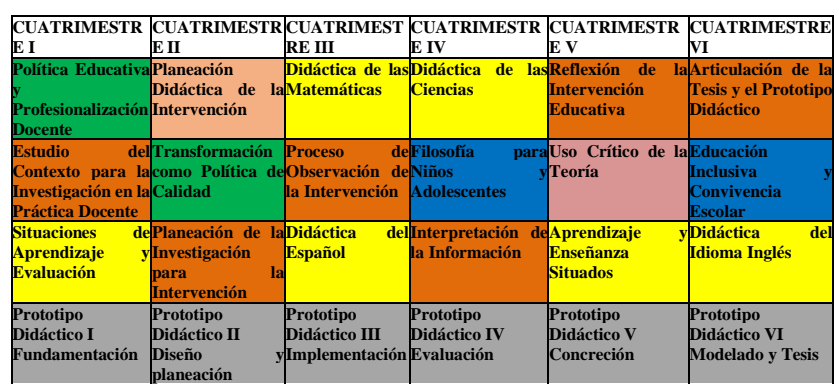

Tabla 13 Malla curricular MIEEB, 2019

A partir del procedimiento de seguimiento a la aplicación de los programas de curso, se observó que la mayoría de los cursos fueron desarrollados sin sufrir modificaciones, cuestión que se considera favorable porque la adecuación de la maestría consistió en un proceso de fortalecimiento de los cursos. Estas valoraciones influyeron en que el programa de la MIEEB se adecuará y no rediseñara, lo que implicó un proceso de menor trabajo intelectual y tiempo con relación al diseño.

En el SGC, el procedimiento: Monitoreo y Evaluación de la Aplicación de la Planificación docente de asignatura o curso tiene como propósito:

Realizar el monitoreo y evaluación a la aplicación de la planificación docente de asignatura y/o curso de Licenciatura y/o Posgrado con base en los criterios establecidos en la plataforma del Sistema de Gestión de la Calidad, para garantizar el logro del perfil de egreso de los estudiantes de las 36 Escuelas Normales Públicas del Estado de México.

El procedimiento tiene sub procedimientos por lo que el responsable elabora un plan de monitoreo para desarrollarlo. Uno de los sub procedimientos es la observación en el aula, para esto, se diseñó un instrumento con el propósito de evidenciar si los elementos del programa están siendo aplicados por el docente, por ejemplo, "se promueve la reflexión a partir de las líneas de discusión”. Los resultados muestran que los aspectos son observados al $100 \%$, y esto se mostró en los informes cuatrimestrales emitidos por el responsable del procedimiento (ver Gráfico 1). 


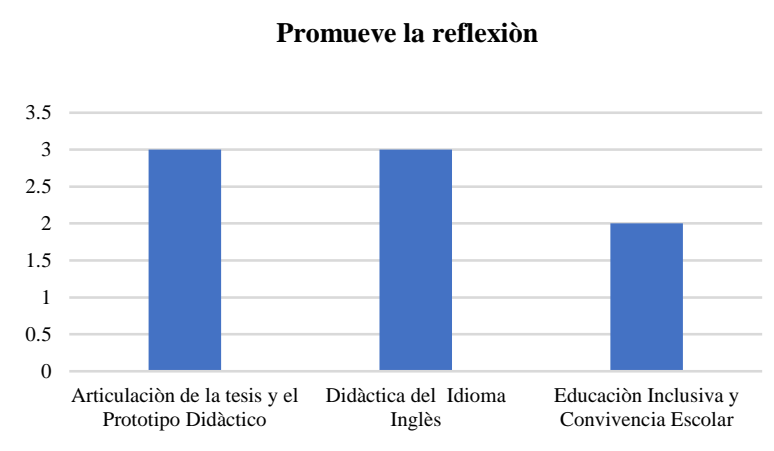

Gráfico 1 Resultados de la observación en el aula

Los resultados muestran que la modalidad didáctica de los cursos está siendo implementada por los docentes que tienen la función de fomentar la reflexión. En el caso del curso Educación Inclusiva se observa una disminución del porcentaje alcanzado y es debido a que fueron menores las sesiones recuperadas, no obstante, el aspecto se observó.

Para evitar caer en excesos de confianza con respecto a los resultados se aplicaron otros instrumentos, se analizan y contrastan para tener mayor información y comprender cómo se implementan los programas y cursos de la maestría, por tanto, se aplican también a los estudiantes, tal es el caso de la encuesta de satisfacción del cliente. En los resultados se muestra que la satisfacción disminuye y se identifican los cursos que tuvieron mayor impacto en su profesionalización. Por ejemplo, los resultados de esta encuesta en el último cuatrimestre, exponen que los alumnos están en mayor porcentaje "muy satisfechos" en Didáctica del Idioma Inglés, de manera progresiva este grado de satisfacción disminuyó en los demás cursos: Articulación de la Tesis y el Prototipo Didáctico; Educación Inclusiva y Convivencia Escolar; y, Prototipo Didáctico VI (ver Gráfico 2).

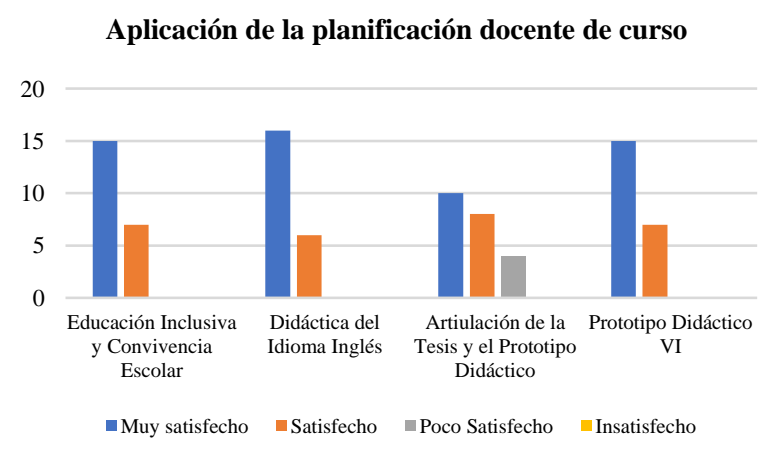

Gráfico 2 Encuesta de satisfacción
La pregunta de esta gráfica se relaciona con la aplicación de la planificación docente y los resultados muestran un mayor grado de satisfacción de los estudiantes en Didáctica del Idioma Inglés, de manera similar en las otras preguntas de la encuesta: ¿Se cumple con los propósitos del curso? ¿Se cumple con el propósito formativo del programa de estudios? ¿El titular mostró dominio del contenido programático del curso? Así, estos resultados muestran el grado de cumplimiento del programa de estudios y de los cursos de la maestría. En algunos cursos se tuvo un porcentaje significativo de "clientes poco satisfechos" e incluso "insatisfechos" que disminuyó con relación a anteriores cuatrimestres. Las causas no fueron solicitadas en la encuesta y éstas pueden observarse en la aplicación del instrumento denominado evaluación de los cursos.

La evaluación de los cursos profundizó en la valoración de los componentes del programa, por ejemplo, el nivel en que las competencias descritas en el curso fueron desarrolladas, el impacto de los contenidos en la profesionalización docente, los contenidos favorecieron la reflexión; con relación a la conducción del docente se solicita valoren si se "fomentó la reflexión de la práctica docente y la teoría", "los productos académicos parciales fueron evaluados y retroalimentados"; al estudiante se le pide que valore su participación, las "aportaciones derivadas de la investigación autónoma", entre otros aspectos.

Los resultados de esta evaluación muestran similitudes con los obtenidos en la encuesta de satisfacción del cliente con relación al impacto de los cursos y al desempeño del docente en la conducción del curso, sobresale la valoración que los estudiantes realizan de su participación la cual está en el indicador de regular. Lo más relevante en este instrumento es el apartado referido a observaciones o sugerencias, ya que en este se pueden identificar algunas debilidades y fortalezas que fueron objeto de análisis en la academia de posgrado (ver Tabla 14). 


\begin{tabular}{|c|c|}
\hline \multicolumn{2}{|c|}{ Observación o sugerencia } \\
\hline Debilidades & Fortalezas \\
\hline $\begin{array}{l}\text { Y hubo temáticas muy } \\
\text { relevantes las cuales se } \\
\text { abordaron en más tiempo } \\
\text { lo cual también estuvimos } \\
\text { sobre el tiempo sin } \\
\text { embargo se trabajó lo } \\
\text { mejor que se pudo. } \\
\text { Hubo momentos en los } \\
\text { que le dimos más } \\
\text { prioridad a identificar la } \\
\text { problematización, } \\
\text { dejando un poco ciertos } \\
\text { contenidos del curso. } \\
\text { El exceso de productos } \\
\text { hace que no se analicen } \\
\text { los temas a profundidad al } \\
\text { igual que las lecturas, } \\
\text { como sugerencia ponerse } \\
\text { de acuerdo al número de } \\
\text { productos o tareas a } \\
\text { entregar. } \\
\text { Implementar estrategias } \\
\text { de enseñanza que } \\
\text { permitan abarcar el tema } \\
\text { planeado totalmente con } \\
\text { la participación activa de } \\
\text { los estudiantes. }\end{array}$ & $\begin{array}{l}\text { El curso brinda las } \\
\text { herramientas para } \\
\text { transformar e intervenir } \\
\text { en la práctica docente. } \\
\text { Durante el desarrollo del } \\
\text { curso se mantiene una } \\
\text { línea de trabajo de } \\
\text { investigación autónoma y } \\
\text { el doctor, emite } \\
\text { sugerencias de } \\
\text { bibliografía a emplear en } \\
\text { el prototipo didáctico } \\
\text { como referentes } \\
\text { sustento de } \\
\text { planteamiento } \\
\text { problema. } \\
\text { La modalidad de trabajo } \\
\text { sugerido por el maestro es } \\
\text { de acuerdo a las } \\
\text { competencias } \\
\text { profesionales que nos } \\
\text { fortalecen y es un buen } \\
\text { momento para aprender } \\
\text { otras opciones para } \\
\text { interactuar en los sistemas } \\
\text { de la tecnología y } \\
\text { modernidad, tal es el caso } \\
\text { del blog del curso que es } \\
\text { muestra de nuestros } \\
\text { alcances. }\end{array}$ \\
\hline
\end{tabular}

Tabla 14 Evaluación de los cursos

Algunas de las observaciones emitidas están relacionadas al tiempo de duración del cuatrimestre el cual fue menor con relación a lo programado debido a las suspensiones derivadas del sismo ocurrido en septiembre de 2017, no obstante, fueron socializados a los docentes y se tomaron acuerdos para disminuirlas y fortalecer la implementación del programa.

El procedimiento denominado la Evaluación del Prototipo Didáctico se valoró a partir de los avances que los estudiantes presentan en la construcción de la Tesis y el Prototipo Didáctico; la evaluación se realiza por el tutor a partir de un instrumento que requisita de cada tutorado y es un elemento que permite llevar un seguimiento del avance de cada estudiante.

La evaluación puede mostrar que todos los aspectos están en proceso, por tanto, lo importante de este instrumento se recuperó en el apartado valoración general (ver Tabla 15).

\section{Valoración general}

V1. El documento se encuentra en estado concluido y avanzado en la mayoría de sus componentes, se realizará la primera fase de acción en el cuarto cuatrimestre.

V2. El documento se encuentra en proceso en los componentes señalados, se solicita al maestrante que concluya los apartados señalados para que implemente la fase de acción en las primeras semanas del cuatrimestre IV.

V3. El documento se encuentra detenido en los componentes señalados como integrados, debido principalmente la falta de lectura e integración de evidencias a la problematización, se le ha solicitado al estudiante que concluya los apartados señalados, sin embargo, su avance es mínimo en cada sesión; existe rezago en este documento de dos cuatrimestres.

Tabla 15 Evaluación del Prototipo Didáctico

Los resultados de esta valoración muestran el rezago en la construcción de la tesis, y se queda como evidencia del seguimiento.

Las causas de este rezago están vinculadas a la modalidad presencial en la que la maestría se implementa, otra es que los estudiantes son docentes de educación básica y tienen carga excesiva de actividades escolares, otros estaban en proceso de evaluación por parte del Sistema Nacional de Evaluación, otra causa es la referida a los años de egreso de la licenciatura algunos tienen más de 20 años que egresaron y otros son recién egresados los que tienen mayor tiempo de egresados presentan mayores debilidades de análisis y redacción.

Algunas causas se atendieron o superaron por los estudiantes y tutores a partir de la tutoría, también se organizaron coloquios cuatrimestrales en los que los comentaristas emitieron observaciones y sugerencias para fortalecer la construcción de las tesis.

Otro instrumento para conocer el avance en la construcción de la Tesis y el Prototipo Didáctico es el aplicado a los estudiantes por el responsable del proceso de monitoreo y consiste que él reporte su avance en términos descriptivos y con un porcentaje (ver Tabla 16). 


\begin{tabular}{|l|r|}
\hline \multicolumn{1}{|c|}{ Avance reportado } & \% de avance \\
\hline Organización de anexos & 95 \\
\hline Fundamentación y esquema general & 90 \\
\hline Trabajando en el primer ciclo de análisis & 80 \\
\hline Modificación de fichero numérico & 75 \\
\hline Fundamentación y esquema general & 70 \\
\hline Estructura general & 90 \\
\hline Informe con soporte teórico & 90 \\
\hline Estructura general del prototipo & 90 \\
\hline Informe general & 80 \\
\hline Estructuración de patente prototipo & 90 \\
\hline Revisión y estructuración de las estrategias & 70 \\
\hline Terminación y análisis del segundo ciclo & 80 \\
\hline Análisis del segundo ciclo de acción & 65 \\
\hline $\begin{array}{l}\text { Análisis y restructuración de ciclo de } \\
\text { acción }\end{array}$ & 70 \\
\hline $\begin{array}{l}\text { Conclusión del análisis del segundo ciclo } \\
\text { de intervención }\end{array}$ & 80 \\
\hline Estructura del segundo ciclo de acción & 70 \\
\hline $\begin{array}{l}\text { Modificación de las conclusiones y } \\
\text { organización de anexos }\end{array}$ & 95 \\
\hline Organización de conclusiones & 95 \\
\hline Análisis del plan de acción & 65 \\
\hline $\begin{array}{l}\text { Reflexión del primer ciclo y análisis del } \\
\text { segundo ciclo }\end{array}$ & 60 \\
\hline
\end{tabular}

Tabla 16 Avance en la construcción de la Tesis y Prototipo Didáctico

Los avances que reportan los estudiantes son poco claros, por ejemplo, la estructura general con un $90 \%$, al igual que quien está fundamentando, otros que están reflexionando el primer ciclo de investigación-acción y una de las causas es que se les dio la posibilidad de reportarlo sin sustentarse en la estructura de la tesis y el prototipo didáctico, y señalar el componente y el avance en este.

De igual manera, los porcentajes de avance reflejan una visión que puede diferir de los que se observen en el documento presentado como borrador, algunos estudiantes tienen un mayor avance, sin embargo, reportaron un porcentaje menor y viceversa. Situación la anterior que se tiene que analizar porque no permite tener información concreta del estado real de las tesis y, por tanto, incidirá en el número de estudiantes que obtengan su grado.

Otro instrumento empleado fue el aplicado en línea a los estudiantes de la maestría para su adecuación que valora la contribución de la maestría en la profesionalización docente; los estudiantes valoran en los niveles de suficiente, poco y no ha influido (ver Tabla 17).

\begin{tabular}{|c|c|c|c|}
\hline $\begin{array}{l}\text { De qué manera ha } \\
\text { contribuido la } \\
\text { MIEEB en los } \\
\text { siguientes aspectos: }\end{array}$ & Suficiente & Poco & $\begin{array}{l}\text { No ha } \\
\text { influido }\end{array}$ \\
\hline $\begin{array}{l}\text { Analizar, reflexionar y } \\
\text { transformar la práctica } \\
\text { educativa atendiendo } \\
\text { la complejidad y el } \\
\text { contexto del trabajo en } \\
\text { la educación básica. }\end{array}$ & $90 \%$ & $10 \%$ & $0 \%$ \\
\hline $\begin{array}{l}\text { Producir } \\
\text { conocimientos para la } \\
\text { mejora continua en la } \\
\text { intervención hacia una } \\
\text { práctica educativa de } \\
\text { calidad a partir de } \\
\text { procesos } \\
\text { investigación. }\end{array}$ & $90 \%$ & $10 \%$ & $0 \%$ \\
\hline $\begin{array}{lr}\text { Desarrollar } & \text { su } \\
\text { identidad profesional y } \\
\text { ética atendiendo } \\
\text { asertiva } \\
\text { creativamente } \\
\text { problemáticas las } \\
\text { necesidades inherentes } \\
\text { a su práctica educativa. }\end{array}$ & $85 \%$ & $15 \%$ & $0 \%$ \\
\hline $\begin{array}{l}\text { Los fundamentos } \\
\text { teóricos, } \\
\text { metodológicos } \\
\text { didácticos } \\
\text { contemplados en los } \\
\text { cursos: didáctica de las } \\
\text { matemáticas, español y } \\
\text { ciencias han } \\
\text { permitido: }\end{array}$ & $90 \%$ & $10 \%$ & $0 \%$ \\
\hline $\begin{array}{lcc}\text { Fortalecer } & & \text { el } \\
\text { conocimiento de } & \text { la } \\
\text { didáctica para } & \text { la } \\
\text { enseñanza y } & \text { el } \\
\text { aprendizaje, en el } \\
\text { diseño de situaciones } \\
\text { de aprendizaje y su } \\
\text { evaluación. }\end{array}$ & $90 \%$ & $10 \%$ & $0 \%$ \\
\hline
\end{tabular}

Tabla 17 Valoración del Programa de Estudios de la MIEEB (Estudio de Factibilidad 2019)

Los resultados muestran que la maestría ha contribuido en el desarrollo de la profesión docente al desarrollar los rasgos del perfil de egreso y las competencias profesionales con un nivel suficiente, la mayoría de los estudiantes valoró como poco y ninguno respondió que no influyó. Por tanto, esta valoración nos brinda información de que al implementarse el programa de estudio y los de cursos, estos están incidiendo, entre otros propósitos a transformar la práctica educativa, desarrollar la identidad profesional y fortalecer la didáctica de los estudiantes. 
Por último, se diseñó el Cuestionario de Pre-egreso para conocer también cómo el programa de estudios impactó en la profesionalización docente a partir de preguntas centradas en los aspectos: académico, perfil de egreso, desempeño de los docentes formadores y como síntesis se plantea la pregunta, ¿cómo calificarías a la Escuela Normal de Ixtlahuaca, en la implementación de este posgrado? (ver Tabla 18).

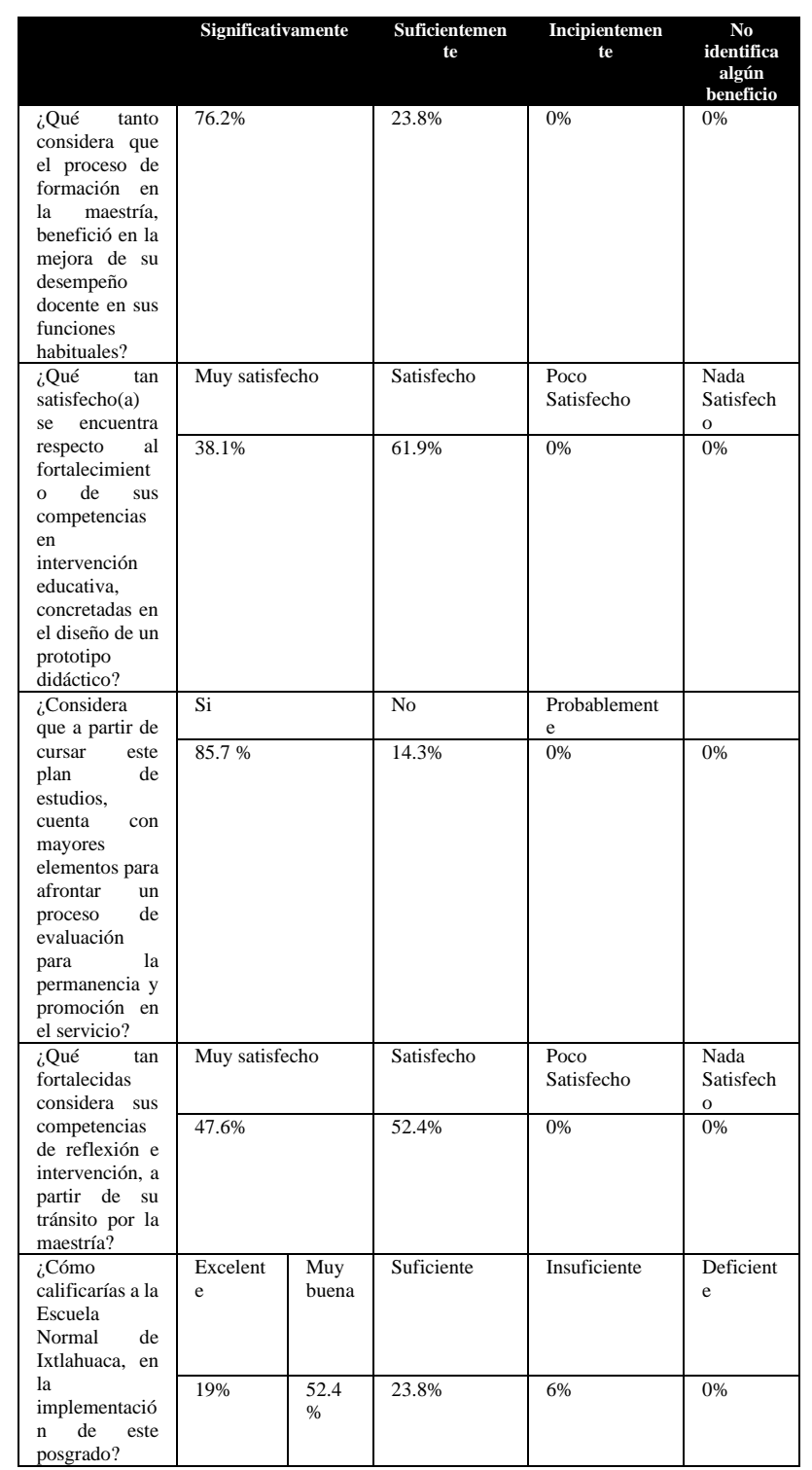

Tabla 18 Cuestionario de Pre-egreso

En términos generales, se puede valorar que la implementación de la maestría benefició significativamente el desempeño docente, se encontró que los estudiantes en mayor porcentaje están satisfechos respecto al fortalecimiento de sus competencias, consideran que tienen mayores elementos para afrontar los procesos de evaluación, tienen fortalecidas sus competencias para la reflexión e intervención.
Consideran que la implementación de la maestría fue muy buena con un porcentaje de $52.4 \%$ y de manera significativa, se obtiene un porcentaje de $6 \%$ que consideró insuficiente este proceso, aspecto que muestra que se existen áreas de oportunidad en una segunda implementación.

\section{Conclusiones}

El seguimiento a los programas de estudio, se realizó a través de diversos procesos, procedimientos e instrumentos, cada uno de los cuales nos brinda información valiosa para realimentar la implementación de la maestría, algunos de los descritos en la presente ponencia podrían considerarse repetitivos, sin embargo, cada uno cumple un propósito porque al triangular la información los resultados nos permiten tener una visión general del proceso y encontrar las debilidades en las que hay que incidir.

Los resultados de estos instrumentos fueron socializados en la academia de posgrado y se tomaron acuerdos para fortalecer los ámbitos de intervención en los que se encontraron debilidades, por ejemplo, los comentarios de los estudiantes en los que consideran que existe un "exceso de productos entregar", "se implementen estrategias de enseñanza", en colegio se acordó: recuperar las orientaciones didácticas y de evaluación de los programas. Aunado a lo anterior, se llegaron a acuerdos concretos en los que los casos así lo requirieron. Con relación a las debilidades que se presenta en la construcción de la tesis, el compromiso es de cada tutor para concluir el proceso con los estudiantes que le fueron asignados para que logren obtener el grado de maestros en intervención educativa.

Con estas valoraciones se constata que aun cuando los instrumentos son diferentes los resultados muestran un impacto favorable del programa estudio y de los cursos en la profesionalización docente y esto dependió en gran medida de los docentes de curso y tutores que estuvieron directamente involucrados en el proceso de implementación y que al igual que la coordinación llevaron su propio seguimiento a la aplicación de su programa y del desempeño de los estudiantes. 
Con relación al impacto del seguimiento a la aplicación de plan y programas de estudio en la profesionalización de los docentes de Educación Básica, se concluye de manera preliminar que, a partir de los resultados aquí analizados, el seguimiento tuvo un impacto favorable porque al efectuarlo desde diversos procedimientos y figuras se constata que se logró el desarrollo de las competencias profesionales y el perfil de egreso de los docentes de educación básica.

\section{Referencias}

CIEES. (2014). Metodología general para la evaluación de programas educativos de nivel superior. México: CIEES

ENI. (2017). Plan de trabajo. Documento interno de trabajo.

SEN. (2017a). Maestría en Intervención Educativa para la Educación Básica. México: SEN.

SEN. (2017b). Seguimiento Académico de los Posgrados que se ofertan en las Escuelas Normales Públicas del Estado de México.

SEN. (2017c). Sistema de Gestión de la Calidad. Consultado en https://enpemsgc2017.wixsite.com/iso9001/iso9001-2015en 